\title{
Analysis and study on static load test of box girder bridge in mining area under special load
}

\author{
Shengtao Yuan, Weilong Zheng, Shunchao Chen*, Chuanwen Hu, Wenbo Luo, Shoufu Xie \\ College of Civil Engineering, Southwest Forestry University, Kunming, Yunnan, 650224, China
}

\begin{abstract}
In order to evaluate the stress condition and working performance of a box girder bridge in a mining area under the special load of 100T, through static load test, the mechanical properties of the bridge under operating conditions were analyzed and studied. Static load test is the stress test of the main beam under the partial and medium load, finally, the measured results are compared with the finite element result. The results show that under the action of various loads, the check coefficient of the bridge strain and the main tensile strain check coefficient was not exceed the standard allowable value 1, the actual working performance of the structure is very good, the maximum relative residual strain was $8.33 \%$, which are not $20 \%$ higher than the standard allowable value, the whole bridge is in the elastic deformation stage, meet with the normal use requirements.
\end{abstract}

\section{Foreword}

Bridges are the throat of transportation, with the rapid development of China's automobile industry and the continuous introduction of foreign cars, as well as the demand for heavy trucks in the mining area and the continuous improvement of production equipment and technology in the mining area, the extra heavy vehicle brings hidden trouble to the safety of bridge in mining area. In order to ensure the normal operation of bridges in the mining area, it is necessary to test the working state of bridge structure through load test and to determine the stress condition of the bridge.

At present, more and more scholars study the static load test of box girder bridge under special load, some research results have been obtained. Such as Huang Jun, Li Shenglian, etc conducted static load test on trestle bridge of hydropower station by loading through water tank[1]; Dong Shuiying conducted an experimental study on a 419.3t special vehicle passing through a hollow slab bridge[2]; Gu Yongjun studied and analyzed the safety of special vehicles crossing the bridge[3]; Su Jiafeng, $\mathrm{Xu}$ Yufeng et al. studied the safety evaluation of wind power generation transportation vehicles passing the simple supported girder bridge[4]; Li Desheng, Zhou Zhaohuan et al studied the load test of transporting wind turbine equipment across the bridge[5]; Liu xiao zhou was analysised of bearing capacity of bridge under special load[6]. However, there is little literature about the load test of box girder bridge under special load in China. Therefore, the research of this subject has application value.

\section{Project summary}

$\mathrm{K} 0+527.81$ middle bridge is located in Shanxin village, line S304 of provincial highway, across the Dianxi river, the superstructure consists of $6 \times 20 \mathrm{~m}$ prestressed concrete box girder, its bridge span connection is simple and continuous; the substructure is composed of ribbed slab abutment, double column abutment and abutment pile foundation. This bridge is $60 \mathrm{~m}$ in length and 3 spans in total, the total width of the bridge is $0.5 \mathrm{~m}+11 \mathrm{~m}+0.5 \mathrm{~m}$, and the beam height is $1.8 \mathrm{~m}$, the bridge deck pavement is composed of $15 \mathrm{~cm}$ thick C50 concrete and inorganic waterproof layer, the transverse slope of the bridge deck is $2 \%$, and the design load is $100 \mathrm{~T}$.

The bridge model is built and analyzed using the bridge software MIDAS/Civil. the test was carried out by simulating a special load of 100T, the stress and displacement of each section of the superstructure are measured under static load, compare and analyze whether the stress and displacement values of the measuring points in each section conform to the design specifications

\section{Bridge static load test}

\subsection{Static load test principle}

Static load test mainly tests the strain, displacement, residual strain and residual deformation after unloading at the measured points of each control section of the upper main structure of the bridge, comprehensive evaluation of bridge main structure performance. The simulated design load of mine $100 \mathrm{~T}$ super heavy vehicle 
is adopted, loading at the most unfavorable position of the internal force influence line in the control section of the main structure, make the test load efficiency coefficient between 0.85 1.05. According to the calculation and analysis, it is determined that the bridge static load test requires two 100T double rear axle heavy vehicles. When the test vehicle is fully loaded, the axle load and wheelbase are as shown in figure 1 .

Strain test: the strain gauge is arranged at the bottom of the box girder and the web of the box girder, the resistance of $120 \Omega$ and the sensitivity coefficient is 2.08 , the range of $10 \mathrm{~cm}$, the data is recovered by DH3819 wireless static strain tester. Deflection test: at the bottom of each control section box girder, the method of suspension wire + hammer + dial indicator is adopted, a hook is set in the middle of the bottom of the beam of the test section, hang the wire on the hook, the hammer is suspended at the bottom of the wire, percentage table under the weight, the weight is constrained by a fixed bracket, as shown in figure 2 .

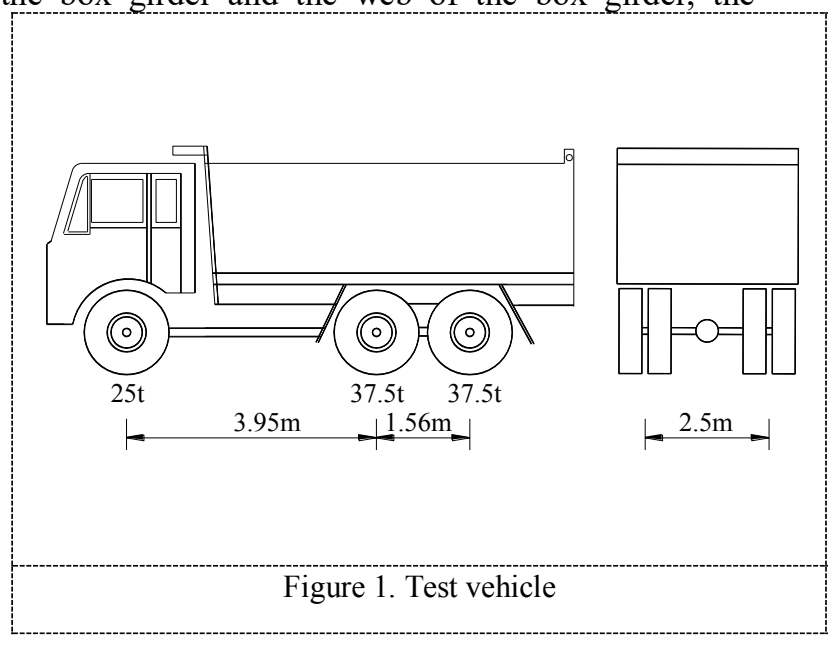

\subsection{Test section and point arrangement}

According to the most unfavorable principle of force, select $1 \#$ span as the test span, it mainly tests the stress and deformation of the main beams in cross sections $\mathrm{J} 1$, $\mathrm{J} 2$ and $\mathrm{J} 3$ of $1 \#$ span, the test section layout is shown in figure 3 , the corresponding strain points are arranged at

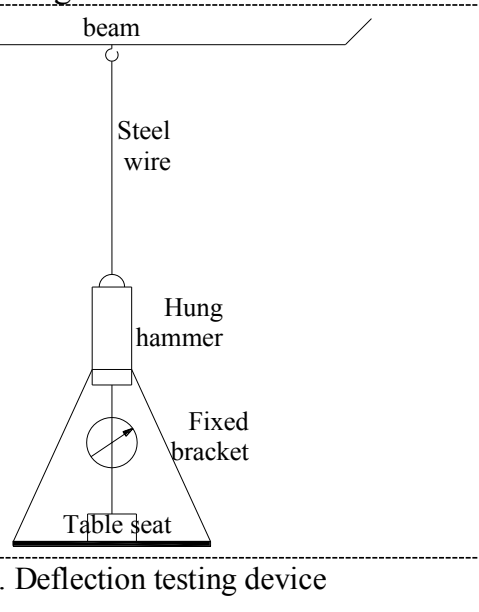

the bottom and side of each control surface beam, in the direction of great mileage, from left to right, 18 strain points are arranged in section $\mathrm{J} 1$ and 12 strain points in section $\mathbf{J} 2$, there is no strain point on the web of section $\mathrm{J} 2$, and 4 strain points are arranged on section $\mathrm{J} 3$ of shear force at beam end.

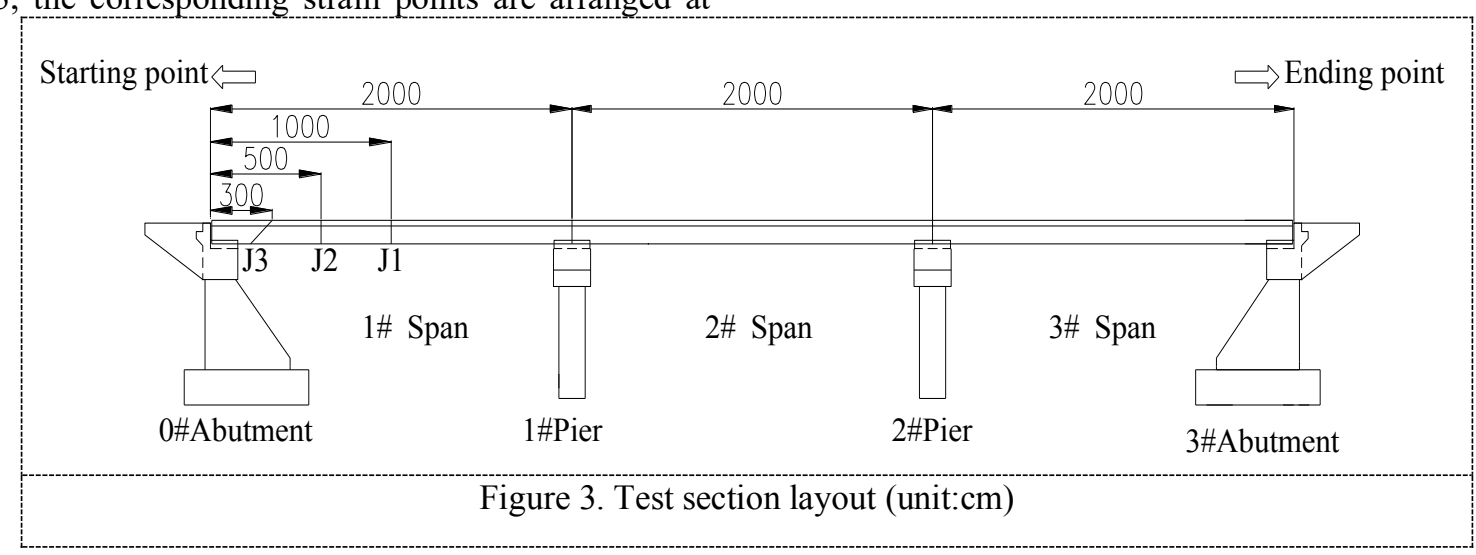

\subsection{Load arrangement and test conditions}

The three sections $\mathrm{J} 1, \mathrm{~J} 2$, and $\mathrm{J} 3$ across $1 \#$ are loaded in turn, 2 cars are placed in the horizontal direction for each section loading car, loading mode is divided into medium load and partial load, the loading truck of each section is arranged in the same way along the longitudinal direction, and the shear section at the beam end is loaded by partial load.

According to the calculation of the structure, the maximum positive bending moment of the control section and the maximum shear force at the beam end are taken as the control values of the loading section, according to the special load $100 \mathrm{~T}$ at the most unfavorable position. the load flow is shown in table 1

Table 1 Load test procedure

\begin{tabular}{cccc}
\hline Load condition & Control section & Loading way & Test content \\
\hline Condition 1 & J1 section & Partial load & Deflection and strain \\
Condition 2 & J1 section & Middle load & Deflection and strain
\end{tabular}




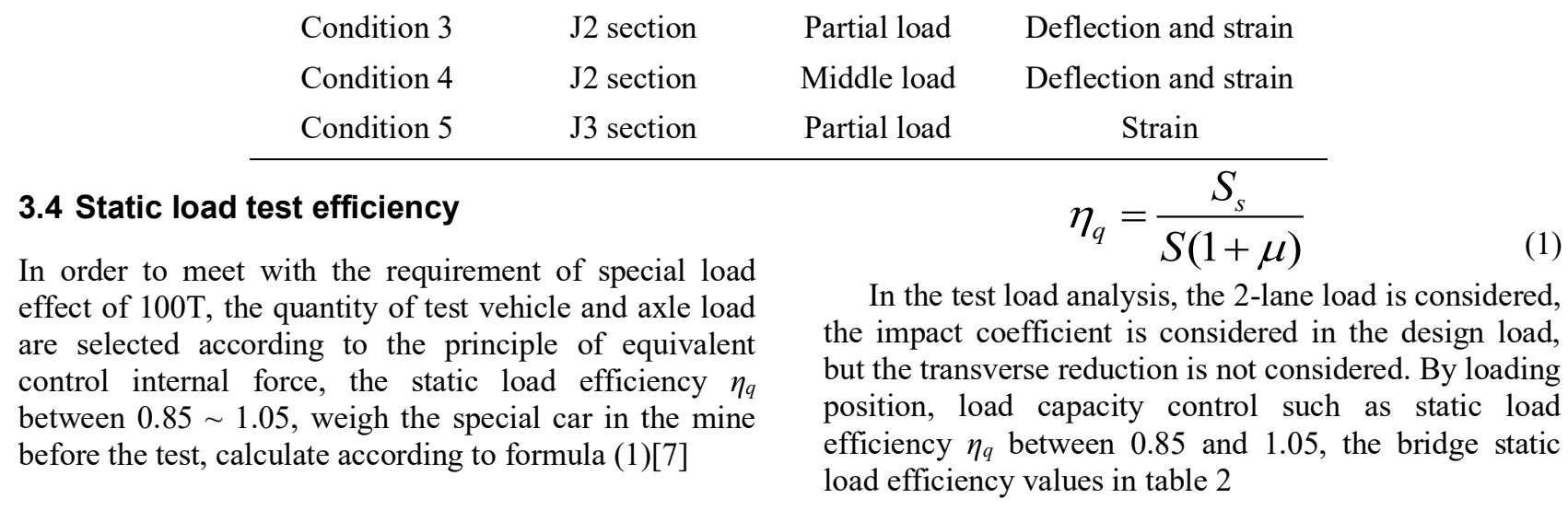

Table 2 Static load efficiency coefficient

\begin{tabular}{cccc}
\hline Load condition & $M S /(\mathrm{kN} . \mathrm{m})$ & $M t /(\mathrm{kN} . \mathrm{m})$ & $\eta_{q}$ \\
\hline Condition 1 & 1377.5 & 1583.22 & 0.87 \\
Condition 2 & 1192.4 & 1339.83 & 0.89 \\
Condition 3 & 990.8 & 1152.06 & 0.86 \\
Condition 4 & 848.3 & 998.03 & 0.85 \\
Condition 5 & $389 \mathrm{kN}$ & $458 \mathrm{kN}$ & 0.85 \\
\hline
\end{tabular}

\section{Analysis of test results}

\subsection{Analysis of strain test results}

Under each working condition, the strain check coefficient of $1 \# \sim 6 \#$ beam measurement point ranges from 0.46 to 0.90 , it shows that the bridge structure and materials meet with the design requirements and the overall performance of the structure is good; the relative residual strain value is less than $8.33 \%$, less than $20 \%$ of the allowable value specified in literature [7], it shows that the overall stiffness of the bridge has a certain margin and the bridge superstructure in the elastic deformation stage. Under the action of stress, there is no abnormality in each test section and the bridge is under normal stress.

According to the result of strain test, the elastic strain and theoretical elastic strain of each test point are drawn as shown in figure4-5

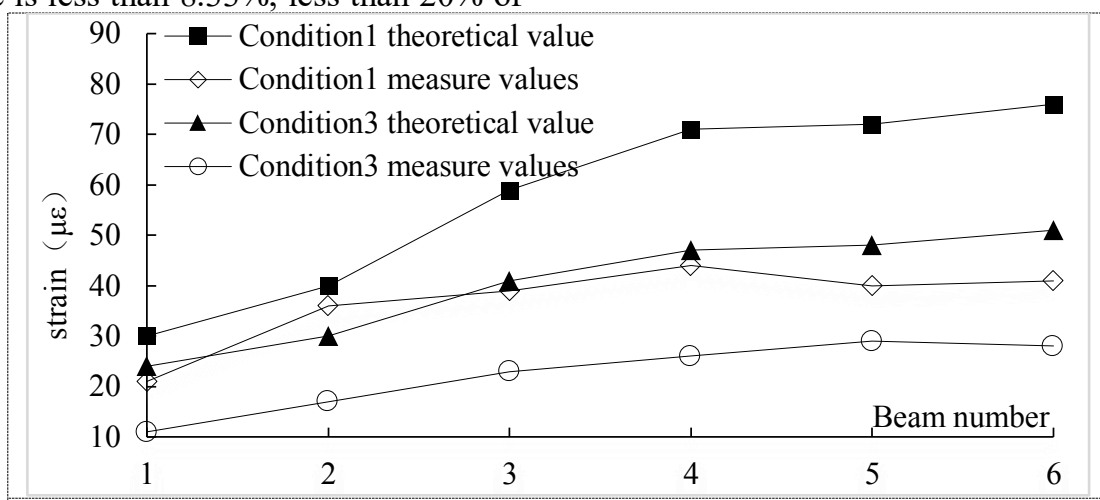

Figure 4. Strain values at working conditions 1 and 3

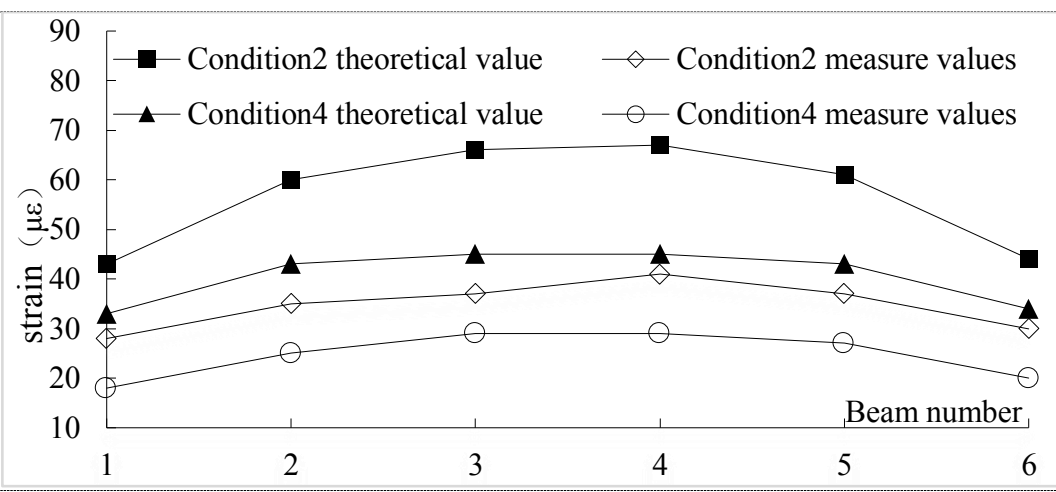

Figure 5. Strain values at working conditions 2 and 4 
As can be seen from figure 4 5, under different loads, the measured strain values of each section are consistent with the calculated values, and the test value is less than the theoretical value, it indicates that the transverse force transfer of the bridge is normal, the overall stiffness distribution of the bridge is relatively uniform.

\subsection{Test results of lateral strain of beam body}

Under test load, the strain data of the web of beam 5 are shown in table 3 .

Table 3 Comparison of measured strain-beam height on the side of beam

\begin{tabular}{cccc}
\hline Beam number & Measuring point position & High measuring point & Measured strain value \\
\hline & Up on left web & 140 & -7 \\
& Middle the left web & 70 & 17 \\
& Under the left web & 20 & 37 \\
$5 \#$ & Average bottom of beam & 0 & 40 \\
& Up on right web & 140 & -5 \\
& Middle the right web & 70 & 16 \\
& Under the right web & 20 & 35 \\
\hline
\end{tabular}

According to the test strain distribution along the beam height, analyze the height and working condition of the $5 \#$ beam neutral axis, as shown in figure6.

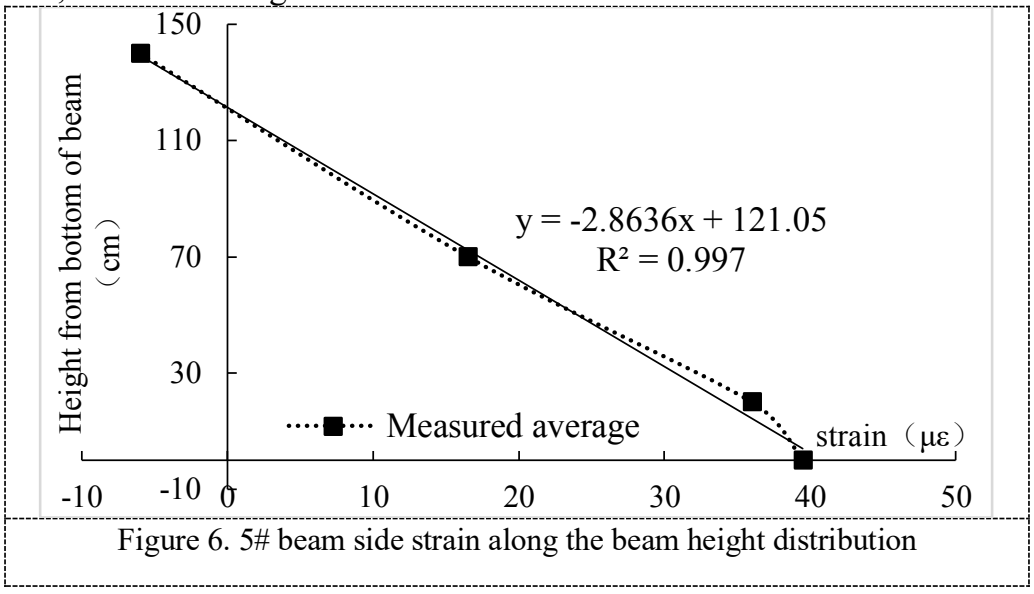

As can be seen from figure 6 , the distribution of the strain value of the $5 \#$ beam test has a good linear relationship along the beam height, the deformation of beam web section conforms to the plane assumption, it indicates that beam 5 is in the elastic deformation stage under vehicle load. According to the linear regression equation, the test neutral axis of no. 5 beam body is $121.05 \mathrm{~cm}$ away from the bottom of the beam, theoretical calculation of the neutral axis height is $99.6 \mathrm{~cm}$, the reason for the deviation is that the bridge deck pavement, the concrete guardrail, etc. are involved in the force, which is a normal phenomenon.

\subsection{Beam end shear section test results}

Under test load, the comparison between the measured values and the calculated values of the main tensile strain at each measuring point of $\mathrm{J} 3$ section $5 \#$ beam is shown in table 4.

Table 4 Comparison between measured main tensile strain value and calculated value

\begin{tabular}{ccccc}
\hline Condition & $\begin{array}{c}\text { Measuring } \\
\text { point number }\end{array}$ & $\begin{array}{c}\text { Main tensile strain } \\
\text { measured value } /(\mu \varepsilon)\end{array}$ & $\begin{array}{c}\text { Main tensile strain } \\
\text { calculation } /(\mu \varepsilon)\end{array}$ & Check coefficient $/ \eta$ \\
\hline Working & $1 \#$ & 3.3 & 5.7 & 0.58 \\
condition & $2 \#$ & 5.1 & 6.2 & 0.82 \\
5 & $3 \#$ & 7.3 & 9.0 & 0.81 \\
& $4 \#$ & 5.2 & 7.4 & 0.70 \\
\hline
\end{tabular}

As can be seen from table 4 , the principal tensile strain check coefficients of the four strain test points in the J3 shear section of beam no.5 are between 0.58 and 0.82 , it meets with the requirement of calibration coefficient $<1$ in literature[7], during the test, visual observation was made near each test section, no visible cracks were found, it shows that the shear bearing capacity of the beam end meets with the design requirements. 


\section{Conclusion}

K0+527.81 middle bridge, under various conditions, static load efficiency is between 0.85 and 0.89 , within the allowed value of $0.85 \sim 1.05$, explaining that the static load test is established, the strain check coefficient is between 0.46 and 0.90 , the principal tensile stress test coefficient is between 0.58 and 0.82 , it indicates that the overall stiffness of the bridge is large and has a certain surplus, beam end shear force meet with design specifications. The distribution of $5 \#$ beam test strain values has a good linear relationship along the beam height, the deformation of beam web section conforms to the plane assumption, the theoretical neutral axis height is less than the measured neutral axis height and overall mechanical performance of the bridge meets with the requirements of normal use

\section{References}

1. Huang.J, Li.L.S, Lin.S.(2010) Static load test for particularly loaded bridge in hydropower station[J]. Technology of Highway and Transport, 2:53-55.
2. Dong.S.Y.(2010) Static calculation and analysis of special load vehicle passing through hollow plate[J]. Technology of Highway and Transport(Applied Technology Edition), 6:288-290

3. Gu.Y.J, S.Y. (2009)Research on reliability evaluation method and technical countermeasures for special load vehicle passing bridge[D]. Hang'zhou:Zhe jiang University of Technology, 2132

4. Su.J.F, Xu.Y.F.(2013)Research on special vehicle safety assessment for existing bridges[J]. Journal of China \& Foreign Highway, 33:230-233

5. Li D.Z, Zhou Z.H. (2012) Analysis of bridge load test with special vehicle load[J]. Urban Roads Bridges \& Flood Control, 1:55-57

6. Liu X.Z. (2009) The analysis of bridge bearing capacity under special load[J]. East China Highway, 177: 53-55.

7. Ministry of Transport of the People's Republic of China, Specification for inspection and evaluation of load-bearing capacity of highway bridges: JTG/TJ21-2011[S] 